\title{
Ph salival y crevicular como método de diagnóstico de la enfermedad periodontal
}

\section{Salivary and crevicular $\mathrm{pH}$ as a diagnostic method for periodontal disease}

\author{
Fabiola Gutierrez-Romero (c) ${ }^{1 \mathrm{a}, \mathrm{b}}$, Liseth Villaverde- Moscol (c)
}

\section{RESUMEN}

La presente revisión de la literatura pretende explicar y entender mejor de manera práctica y clínica la relación del $\mathrm{Ph}$ salival y el $\mathrm{Ph}$ crevicular gingival como una alternativa de diagnóstico precoz rápido en los pacientes con periodontitis crónica y gingivitis.

La relación entre el progreso de la enfermedad periodontal y el fluido crevicular gingival es muy cercana, ya que el desarrollo de la periodontitis está asociado a un incremento en el $\mathrm{Ph}$ del líquido crevicular gingival.

La saliva ha sido empleada en la detección temprana, valoración de riesgo, diagnóstico, pronóstico y seguimiento de la progresión de las diferentes enfermedades bucales. En la detección de marcadores oncológicos como: cáncer de pulmón y riñón así como en enfermedades infecciosas e inmunes.

Palabras claves: Saliva; Periodontitis; Fluido Crevicular Gingival. (Fuente: DeCS BIREME)

\section{ABSTRACT}

This review of the literature aims to explain and better understand in a practical and clinical way the relationship of salivary $\mathrm{Ph}$ and crevicular gingival $\mathrm{Ph}$ as an alternative of rapid early diagnosis in patients with chronic periodontitis and gingivitis.

The relationship between the progression of periodontal disease and the gingival crevicular fluid is very close, since the development of periodontitis is associated with an increase in the $\mathrm{Ph}$ of the gingival crevicular fluid. Saliva has been used in the early detection, risk assessment, diagnosis, prognosis and follow-up of the progression of different oral diseases. The detection of cancer markers such as lung and kidney cancer as well as in infectious and immune diseases.

Key words: Saliva; Periodontitis; Gingival Crevicular Fluid. (Source: MeSH NLM)

${ }^{1}$ Universidad de San Martin de Porres. Maestría en Periodoncia. Lima, Perú

${ }^{a}$ Docente, Maestro en Periodoncia

Este es un artículo Open Access distribuido bajo

${ }^{b}$ Cirujano dentista, estudiante de posgrado. la licencia Creative Commons Atribución-No

Correspondencia:

Fabiola Gutierrez-Romero

Direccion:Av. San Luis 1265, San Luis. Lima Perú.

Correo electrónico: fabiolagutierrezromero@yahoo.com.pe 


\section{INTRODUCCION}

La periodontitis crónica es una enfermedad infecciosa que es resultado de la inflamación de los tejidos blandos y duros que rodean al diente y su destrucción progresiva del tejido conectivo, epitelial y del hueso alveolar. De las patologías periodontales, es la que con más frecuencia se encuentra en la mayoría de los peruanos, al menos 95 de cada 100 personas la padecen.

El diagnóstico de la enfermedad periodontal se realiza mediante parámetros clínicos como: índice de placa, índice gingival, la profundidad de sondaje y nivel de adherencia clínica. La aplicación de métodos de diagnóstico rápidos simples basados en el análisis de la saliva total y el líquido crevicular gingival nos proporciona información sobre la respuesta inmune celular y humoral del paciente, facilitando una detección precoz de los pacientes de riesgo y poder instaurar el tratamiento adecuado en fases tempranas de la enfermedad periodontal.

Existen escasos datos de estudios acerca de la relación patológica de la enfermedad periodontal y la saliva, siendo ésta el lugar dónde drena el líquido crevicular gingival y sus diversos componentes. De este modo es factible creer que en la enfermedad periodontal se modifica muchas propiedades de la saliva y liquido crevicular gingival, tales como su composición y ph ${ }^{(1)}$.

El factor de riesgo ambiental más significativo para la enfermedad periodontal lo constituyen las bacterias que residen en la biopelicula de la placa. En esta patología, la presencia de placa aumenta el flujo del líquido crevicular gingival, favorece la salida de leucocitos polimorfonucleares hacia el surco gingival e inicia la cascada de la inflamación. Por esta razón, el objetivo del tratamiento periodontal es desorganizar la microbiota supragingival e subgingival del cálculo dental alojado en las bolsas periodontales. Los microorganismos patógenos deben proliferar en un medio apto para éstos. Por tal motivo, en la enfermedad periodontal, a medida que aumenta la severidad, favorecerá la reproducción de los periodontopatógenos responsables.

Las bacterias sacarolíticas son las principales colonizadoras en la bolsa periodontal y responsables de la destrucción periodontal como: Fusobacterium nucleatum y Prevotella intermedia. Estos tipos de bacterias se alimentan de proteínas cuyo subproducto es el amoniaco $^{(2)}$.

\section{Saliva y liquido crevicular}

La saliva es propuesta como herramienta diagnóstica para la supervisión del progreso de la enfermedad y la salud buco-periodontal. Los marcadores de la saliva pueden aplicar para la temprana detección, valoración de riesgo, diagnóstico, pronóstico y seguimiento de la progresión de las diferentes enfermedades bucales. Existen también marcadores oncológicos para el cáncer de pulmón y riñón, enfermedades infecciosas e inmunes ${ }^{(3)}$.

La saliva es una secreción exocrina compleja, importante en el mantenimiento de la homeostasis de la cavidad bucal. Es bien conocido que las funciones de la saliva son, en relación con el flujo y la composición molecular (proteínas,glucoproteínas y fosfoproteínas), es el de proteger los tejidos bucales contra la desecación y agresiones del medio ambiente como el de modular los procesos de desmineralización-re mineralización y el de lubricar las superficies dentarias como mantener el balance microbiológico. Se considera que el papel que juega la saliva contra la caries dental es principalmente por su velocidad y cantidad de flujo de este fluido. Si estos mecanismos están alterados pueden causar un desbalance en la limpieza de sustratos bacterianos y en la protección de las superficies dentarias, alterando así la capacidad amortiguadora de la saliva. 
Esta función es importante ya que regula las sustancias que incrementan el ph salival y a los agentes antimicrobianos que existen en el medio bucal; potenciadonse en una enfermedad periodontal ${ }^{(4)}$.

En el surco o hendidura gingival, se encuentra el fluido gingival crevicular, que rodea a los tejidos blandos entornos al diente. En individuos sanos esta hendidura es menor de $0.5 \mathrm{~mm}$ de profundidad. Si excede de este tamaño pasa a denominarse bolsa o bolsillo gingival. Aparece ante inflamación del epitelio de unión que liberaría linfocitos y neutrófilos a la hendidura.

Uno de los mecanismos del huésped para controlar las bacterias en el surco gingival es a través del líquido crevicular gingival. La salida de este exudado ayuda a eliminar los microorganismos no adheridos del espacio dentogingival y a reducir las concentraciones de sus toxinas y productos metabólicos. Por consiguiente, no resulta sorprendentemente el aislamiento de bacterias del líquido crevicular gingival. Este líquido también contiene una serie de componentes antimicrobianos y enzimas que son enviados directamente al área donde se ha producido la colonización bacteriana. El líquido crevicular gingival es rico en elementos celulares aparte de las bacterias. Es frecuente el hallazgo de células originadas en el epitelio de unión y epitelio sulcular oral debido a la renovación constante, este rápido recambio celular parece ayudar a eliminar las bacterias ${ }^{(5-7)}$.

La recolección y análisis del líquido crevicular gingival, se han ido desarrollando con el objetivo de establecer un método simple para determinar la presencia de enfermedad periodontal a través del análisis de los diferentes componentes como mediadores de la inflamación y el ph ${ }^{(8)}$.

\section{PH Salival y ph del líquido crevicular}

Establecer un nuevo método de control de medición cuantitativa a través del $\mathrm{pH}$ salival y
$\mathrm{pH}$ del líquido crevicular gingival en la progresión de la enfermedad periodontal nos serviría de ayuda en la fase de diagnóstico y mantenimiento de los pacientes que sufren enfermedad periodontal. La relación entre el progreso de la enfermedad periodontal y el fluido crevicular gingival es muy cercana, ya que el desarrollo de la periodontitis está asociado con el incremento en el $\mathrm{pH}$ del líquido crevicular gingival a un $\mathrm{pH} 8.5$

El término $\mathrm{pH}$ se utiliza para expresar la concentración de iones hidrogeniones de una solución. Las concentraciones altas de hidrogeniones corresponden a $\mathrm{pH}$ bajos y las concentraciones bajas a $\mathrm{pH}$ altos.

$\mathrm{El} \mathrm{pH}$ se mide en unidades potencio métricas en una escala que va de 0 a 14 . Existen sistemas capaces de controlar los cambios de $\mathrm{pH}$, estos se denominan sistemas de tampón o Buffer. Un sistema de tapón es una solución que contiene dos o más compuestos químicos capaces de prevenir cambios importantes de la concentración de hidrogeniones, cuando se añade un ácido o una base a la solución. Los fluidos intracelulares y extracelulares de los organismos vivos contienen pares conjugados ácido- básico los cuales actúan como tapones del $\mathrm{pH}$ normal de dichos fluidos. El principal tapón extracelular de los vertebrados es el sistema tapón del bicarbonato.

De acuerdo a lo señalado por Skoog, G (1984), existen tres métodos para la medición del $\mathrm{pH}$ en una sustancia liquida.

- A través de cintas

- Medición de Ph con electrodo

- Potenciometro

\section{- $\quad$ Medicion de Ph a travésde cintas}

Las cintas reactivas para medir $\mathrm{pH}$ pueden variar de 1 a 14, pero esto va a depender de la marca comercial. El principio para la medición de $\mathrm{pH}$ se fundamenta en lo siguiente: las tiras son impregnadas con dos indicadores: uno ácido, generalmente rojo fenol y uno alcalino verde de 
bromocresol. Dicho indicadores a $\mathrm{pH}$ neutro son por lo general a color amarillo. En presencia de una solución ácida el indicador cambia a rojo, siendo la intensidad del color inversamente proporcional a las unidades de $\mathrm{pH}$, en presencia de una solución alcalina, el indicador cambiara a tonalidades que varían de verde claro al azul intenso por lo que el color que toma el indicador es directamente proporcional al $\mathrm{pH}$.
De esta manera, al impregnar la cinta reactiva con una solución, puede haber una pequeña perdida de indicador, por lo tanto, el $\mathrm{pH}$ obtenido con esta es aproximado y su uso limitado. No debe ser empleado en exámenes que requieran de un valor de $\mathrm{pH}$ exacto. (Figura 2).

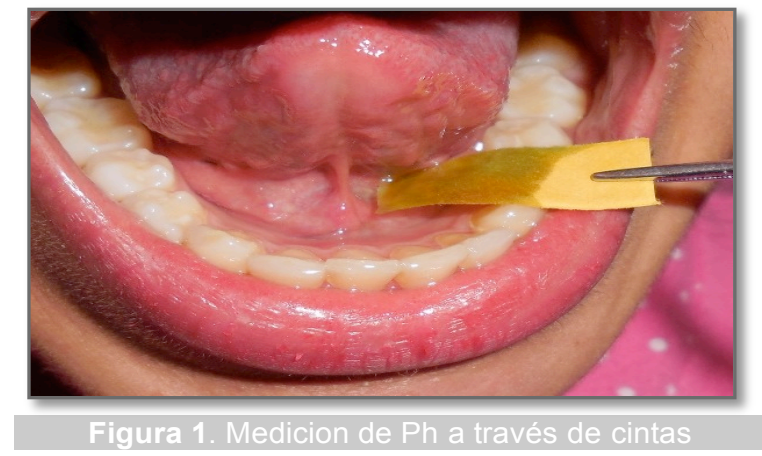

\section{Medición de pH por electrodo.}

Se realiza a través de electrodos de vidrio. Consiste en un par de estos, de fabricación comercial, uno de color y otro sumergido en la solución cuyo $\mathrm{pH}$ se desea medir. Se fabrica el electrodo de vidrio sellando un bulbo de vidrio delgado y sensible al $\mathrm{pH}$, al extremo de un tubo de vidrio de paredes gruesas se llena el bulbo con una solución de ácido clorhídrico saturado con cloruro de plata, se sumerge un alambre de plata en la solución que se conecta a través de un cable de externo a un terminal de un dispositivo para la medida de $\mathrm{pH}$. Se conecta entonces el electrodo de color a la otra terminal y se procede a medir el $\mathrm{pH}$ de la solución.

\section{Potenciómetro}

Existe en el mercado una gran cantidad de medidores de $\mathrm{pH}$ de lectura directa, estos circuitos son relativamente simples donde normalmente tienen dos calibraciones: unidades de $\mathrm{pH}$ y mili volts. Las escalas de unidades de $\mathrm{pH}$ abarcan unos intervalos de 0 a 14 unidades de $\mathrm{pH}$ con un margen de error de +/- 0,02 a +/$0,03 \mathrm{U} / \mathrm{pH}^{(9)}$.

\section{Relación entre ph salival y fluido crevicular con la enfermedad periodontal}

El espacio dentogingival puede actuar como puerta de entrada para los patógenos bacterianos hacia los tejidos periodontales más profundos; no obstante, también constituye una vía de salida para los diversos componentes del líquido crevicular gingival.

La presencia de placa aumenta el flujo del líquido crevicular, favorece la salida de los leucocitos polimorfonucleares hacia el surco, 
gingival e inicia la cascada inflamatoria del huésped. Existen numerosos mediadores de inflamación interlucina1, el factor de necrosis tumoral, la interlucina 8, componentes bacterianos y el $\mathrm{pH}$.

La saliva es el principal protector de los tejidos duros y blandos de la cavidad bucal, suministra iones que intervienen en la remineralización del esmalte, además puede servir como receptora para el inicio de la colonización bacteriana que da origen a la placa.

Existen estudios que relacionan estas dos variables, se ha observado que las personas con producción rápida de cálculo dental también secretan saliva con mayor cantidad de urea. La descomposición de la urea produce amoniaco y este puede aumentar el $\mathrm{pH}$ de la placa.

Cuando existe salud periodontal, el fluido crevicular gingival se comporta como un componente acuoso exudativo que lleva todas las moléculas principales (componentes del complemento y anticuerpos), elementos celulares(neutrófilos y células plasmáticas) y las células responsables que son necesarias para prevenir una invasión del tejido mediante la placa bacteriana hallada subgingival. Este fluido es afectado en su organización y composición, cuando las bacterias periodontopatógenas empiezan a liberar subproductos como el amoniaco.

La relación entre el progreso de la enfermedad periodontal y el fluido crevicular gingival es muy cercana, ya que el desarrollo de la periodontitis es asociado con el incremento en el $\mathrm{pH}$ del líquido crevicular gingival a un $\mathrm{pH} 8.5$ y es pensando que ocurre por la degradación bacterial de las proteínas contenidas en el líquido crevicular gingival.

Se considera que el fluido cervicular y la saliva podrían actuar como biomarcadores de la enfermedad periodontal ${ }^{(10)}$, en particular la saliva, puede considerarse una fuente valiosa de información porque contiene biomarcadores específicos que reflejan la condición de los tejidos en la enfermedades periodontal ${ }^{(11)}$.

Las bacterias sacarolíticas subgingivales utilizan proteínas como fuente principal de alimentación y produce amoniaco como subproducto, cuyo resultado es un incremento del $\mathrm{pH}$ y sirve para el desarrollo de la precipitación de sales de calcio de la saliva o del líquido crevicular gingival, resultando así la formación de cálculo subgingival o directamente ubicado en margen gingival libre.(Figura 2)

En la nueva clasificacion de las Enfermedades Periodontales y Peri implantarias se propone a futuro poder incluir la deteccion de biomarcadores a traves de la saliva y el líquido crevicular para tener un diágnostico mas certero al momento de la definicion de un caso de periodontitis asi como la detección precoz tanto del grado y estadio de la enfermedad, a traves de la saliva se podria identificar restos de ADN de los periodontopatogenos y poder establecer el grado de la periodontitis ${ }^{(12,13)}$.

\section{CONCLUSIONES}

- La elevación del Ph del líquido crevicular gingival y salival se debe a la degradación de las proteínas por parte de las bacterias que se encuentran en el surco gingival.

- El incremento del Ph del líquido crevicular gingival $\mathrm{o}$ del $\mathrm{Ph}$ salival produce la precipitación de las sales de calcio resultando así en la formación de cálculo subgingival.

- Con la medición del Ph salival y del ph liquido crevicular gingival se busca reducir el tiempo de la cita en el diagnóstico y mantenimiento del tratamiento periodontal.

- La saliva podría ser un método de diagnóstico precóz del grado de la periodontitis mediante la identificacion de biomarcadores inflamatorios. 


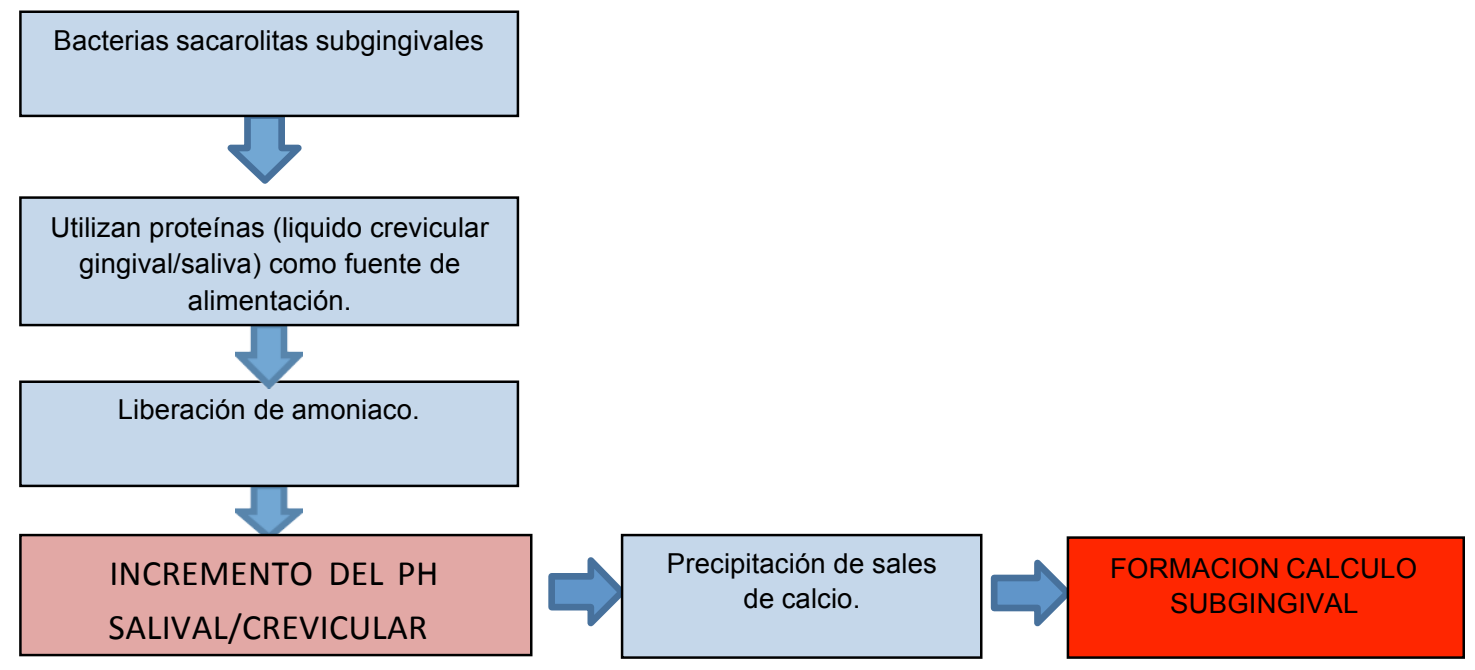

Contribuciones de autoría: FGR y LVM recolectaron y analizaron la información, redactaron y revisaron el articulo. Amabas autoras aprobaron el manuscrito.

Fuente de financiamiento: autofinanciado

Conflictos de interés: no hubo ningún conflicto de interés.

\section{REFERENCIAS}

1. Frias MC, Herrera JI,Carasol M, Donate E. Diagnóstico de la enfermedad periodontal basado en la respuesta del huésped. Cient.dent.2007, 4(2):159-169.

2. Barros S P, Williams R. Gingival crevicular fluid as a source of biomarkers for periodontitis. Periodontol 2000. 2016 Feb; 70(1): 53-64.

3. Guenstsh A, Kramesberger M, Sroka A, Pfister W, Potempa K, Eick S, Comparison of Gingival Crevicular Fluid Sampling Methods in Patients with Severe Chronic Periodontitis. J Periodontol. 2011; 82:10511060

4. Gary C. Analysis of gingival crevice fluid and risk of progression of periodontitis. Periodontol 2000,2004; 34: 109-119.

5. Botero J. Respuesta inmune en las enfermedades del periodonto: desde salud hasta enfermedad y sus implicanciones terapéuticas. Rev. Fac. Odontol Univ Antioq 2009; 2(1): 122-128.

6. Cimansoni G. Crevicular fluid update. Monogr Oral Sci. 1983; 12: III-VII, 1-152.

7. Faria R, Belen A, Bascones A. Nuevos métodos de diagnóstico en Periodoncia Metodos Bioquimicos. Av Periodonton Implantol 2001; 13, 1: 29-37.

8. Romero H.M, Hernández Y. Modificaciones del $\mathrm{pH}$ salival con el uso de aparatología. Revista Latinoamericana de ortodoncia y ortopedia, marzo, 2009.

9. García S, Bravo F, Ayala J, Bardales G. Ph en saliva total en pacientes con enfermedad periodontal del Servicio de Periodoncia de la Facultad de Odontología de la UNMSM. Odontol. Sanmarquina 2008; 11(1): 19-21.

10. González-Ramírez J, Serafín-Higuera N, Silva Mancilla M, Martínez-Coronilla $G$, Famanía-Bustamante $\mathrm{J}$, López López A. Use of Biomarkers for the Diagnosis of Periodontitis,Periodontal Disease Diagnostic and Adjunctive Non-surgical Considerations, Nermin Mohammed Ahmed Yussif, IntechOpen. 2019. DOI: 10.5772/intechopen.85394. Available from: https://www.intechopen.com/books/periodon tal-disease-diagnostic-and-adjunctive-nonsurgical-considerations/use-of-biomarkersfor-the-diagnosis-of-periodontitis 
11. Stepan Podzimek, Lucie Vondrackova, Jana Duskova,Tatjana Janatova, and Zdenek Broukal. Review Article Salivary Markers for Periodontal and General Diseases. Hindawi Publishing Corporation. Disease Markers. Volume 2016, Article ID 9179632, 8 pages. http://dx.doi.org/10.1155/2016/9179632.

12. Tonetti M, Greenwell H, Kornman K. Staging and grading of periodontitis: Framework and proposal of a new classification case definition. Journal of clinical Periodontology. 2017 World Workshop. Juanary 2018.

13. Kim E-H, Joo J-Y, Lee YJ, Koh J-K, Choi J$H$, Shin $Y$, et al. (2018) Grading system for periodontitis by analyzing levels of periodontal pathogens in saliva. PLoS ONE 13(11): e0200900.

Fabiola Gutierrez Romero

ORCID iD: (i) http://orcid.org/0000--0002--1929-0306

fabiolagutierrezromero@yahoo.com.pe

Liseth Villaverde Moscol

ORCID iD: (ㄴ) https://orcid.org/0000-0003-3400-2648

lizeth_villaverde@usmp.pe 